\title{
Preparation of Lotus Nanofibers-Alginate Porous Membranes for Biomedical Applications
}

\author{
Jinfeng Zhang, ${ }^{\mathrm{a}}$ Guangting Han, ${ }^{\mathrm{b}, *}$ Yuanming Zhang, ${ }^{\mathrm{b}}$ Ying Gong, ${ }^{\mathrm{c}}$ and Wei Jiang ${ }^{\mathrm{b}, \mathrm{d}}$
}

Tissue and organ failure or loss is a major problem for human health, and the construction of tissue engineering porous scaffold materials is a core step in the repair of damaged tissue and organs. Fibers from the lotus (Nelumbo nucifera) plant can be a source of superfine fibers. Such fibers have excellent biocompatibility, and they are easy to convert into nanofibers that could be applied for tissue engineering. Lotus fibers were carboxyl-modified with the TEMPO/NaClO/NaBr system, and lotus nanofibers were prepared. The effect of oxidation conditions on their morphologies and degrees of oxidation were investigated. The diameters of the lotus nanofibers were about $15 \mathrm{~nm}$. Additionally, the degrees of oxidation of lotus nanofibers increased with an increase of oxidation time. Moreover, the oxidized sodium alginate membranes crosslinked with carboxymethyl chitosan degraded quickly, with the degradation rates increased by $82.1 \%$ or $100.0 \%$ during a 14 -day period. By increasing the dosage of carboxymethyl chitosan, the $\mathrm{pH}$ values tend to rise. In comparison, the degradation rates of the lotus nanofibers/alginate porous membranes crosslinked with carboxymethyl chitosan exhibited better performance in terms of microstructure, porosity, water absorption, mechanical properties (0.36 MPa /7.7\%), and in vitro degradation (59.2\%).

Keywords: Lotus fibers; Polymer; Nanofibers; Degradation; Porous membranes

Contact information: a: Department of Surgery, Songshan Hospital of Qingdao University, Qingdao 266021, Shandong, China; b: State Key Laboratory of Bio-Fibers and Eco-Textiles; Collaborative Innovation Center for Eco-Textiles of Shandong Province, Qingdao University, Qingdao 266071, Shandong, China; c: Department of Pathogenic Biology, School of Basic Medicine, Qingdao University, Qingdao 266021, Shandong, China; d: College of Textiles, Qingdao University, Qingdao 266071, Shandong, China;

*Corresponding author: kychgt@qdu.edu.cn.

\section{INTRODUCTION}

Lotus fibers located in the vascular bundle of lotus petioles exhibit a spiral shape. They are composed of cellulose (up to $41.3 \%$ ), hemicellulose, and lignin. The diameters of lotus fibers are approximately $3 \mu \mathrm{m}$, and the diameters of microfibrils are about $5 \mathrm{~nm}$, which are advantages for the easy preparation of lotus nanofibers (Pan et al. 2011).

However, little research has been done on the degrading performance of new oxidized cellulose-lotus nanofibers. Biodegradable biomaterials show enlarged pore size, porosity, and interconnection of pores over time, but non-biodegradable materials (such as metal material) facilitate the growth of fibrous connective tissues in pores, which can continuously attach to proteins in a non-specific manner, resulting in smaller pores. In general, non-biodegradable materials are averse to cell growth.

In vitro degradation experiments of oxidized cellulose have demonstrated a decrease in mass by $90 \%$ after immersing an oxidized cellulose film with $20.3 \%$ degree of oxidation (DO) in PBS (phosphate buffer solution) for four days (Khil et al. 2005). 
However, the degradation rate of oxidized bacterial cellulose is four times larger than that of a non-oxidized specimen in the same conditions (Peng et al. 2012). Hence, the current research has mainly investigated nanofibers (NF) with a degree of oxidation (DO) smaller than $10 \%$. Furthermore, the influence of the oxidization time on the DO of NF has been analyzed using a calcium acetate gradient (Wu et al. 2012). Carboxyl in the fiber participates in the replacement reaction with calcium acetate. The free carboxylic acid produced can be titrated using a standard sodium hydroxide solution, following Eq. 1.

$$
2 \mathrm{R}-(\mathrm{COOH})+\left(\mathrm{CH}_{3} \mathrm{COO}\right)_{2} \mathrm{Ca} \rightarrow \mathrm{R}-(\mathrm{COO})_{2} \mathrm{Ca}+2 \mathrm{CH}_{3} \mathrm{COOH}
$$

In addition, NFs immersed in PBS buffer ( $\mathrm{pH}$ 7.4) exhibit changes in their degradation rates. The $\mathrm{pH}$ value in the degradation fluid and the FTIR spectrogram are used to analyze the in vitro degradation of NF.

Alginate porous membranes are prepared via physical or chemical crosslinking (Hennink and Van Nostrum 2012). In physical crosslinking, the three-dimensional structure is affected by varying environmental stimuli (e.g., $\mathrm{pH}$, temperature, and ionic strength) or by physical and chemical interactions, such as hydrophobic interactions and charge condensation. In the physical crosslinking of sodium alginate, calcium chloride is commonly used for ionic crosslinking (Gong et al. 2012). Crosslinking reactions between polymer chains form a three-dimensional network structure through the formation of chemical bonds (Rowland et al. 2013).

Alginate porous membranes are produced by covalently crosslinking dihydrazino or diamine with sodium alginate (Bidarra et al. 2014). Crosslinking agents include hexanediol dihydrazide and polyethylene glycol-diamine (Souguir et al. 2012). Through dehydration and the condensation of amino and carboxy, an amide bond can be produced to obtain stable chemically-crosslinked porous membranes. Thus, chemical crosslinking agents can be used to prepare porous membranes with excellent mechanical properties (Bidarra et al. 2014). Unfortunately, most crosslinking agent molecules are toxic and cannot be completely removed from the resulting membrane (Pawar and Edgar 2012).

The degradation rate of sodium alginate is relatively low, and the degradation of gels formed by ionic crosslinking is uncontrollable (Lee and Mooney 2012). The degradation of sodium alginate requires 3 to 6 months to complete. Hence, applications of sodium alginate as scaffolds for tissue engineering have been limited. Thus, sodium alginate needs to be modified. Highly oxidized sodium alginate (OSA) with a degree of oxidation of $50 \%$ can obviously depress cells at a high concentration. At $200 \mu \mathrm{g} / \mathrm{mL}$ concentration, cell depression is strongest, and cytotoxicity reaches level 2 . OSA (DO $\leq$ $30 \%$ ) cannot significantly depress cells at any concentration gradient, and cytotoxicity is less significant and remains at level 0 or 1 . Oxidized sodium alginate with low DO is nontoxic (Gao et al. 2011). Thus, OSA can be used as biological membranes. For the practical use of sodium alginate, this study focused on OSA (DO $\leq 10 \%$ ).

After sodium periodate oxidation, sodium alginate presents two aldehyde groups, which show higher reactivity than $-\mathrm{OH}$ and $-\mathrm{COOH}$. The two aldehyde groups facilitate the Schiff base $(-\mathrm{CH}=\mathrm{N}-)$ crosslinking between sodium alginate and substances with amino groups, such as carboxymethyl chitosan (Figs. 1 and 2). In vivo biodegradation of carboxymethyl chitosan mainly occurs via enzymolysis (Pantaleone et al. 1992). Adjusting the ratio between two polymers can improve the mechanical properties and degradation rate of the mixtures. In this study, O-carboxymethyl chitosan (CMC) with a 90\% degree of substitution was used for the chemical crosslinking of oxidized sodium alginate porous membranes (COSA). Additionally, lotus nanofibers/alginate porous membranes were 
prepared. The degradation performance in vitro was investigated. Lotus nanofibers/ alginate porous membranes maybe an ideal material as tissue engineering scaffolds in the future.

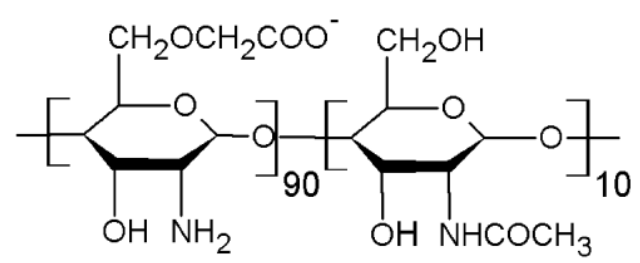

Fig. 1. Molecular structure of O-carboxymethyl chitosan (degree of substitution as $90 \%$ )

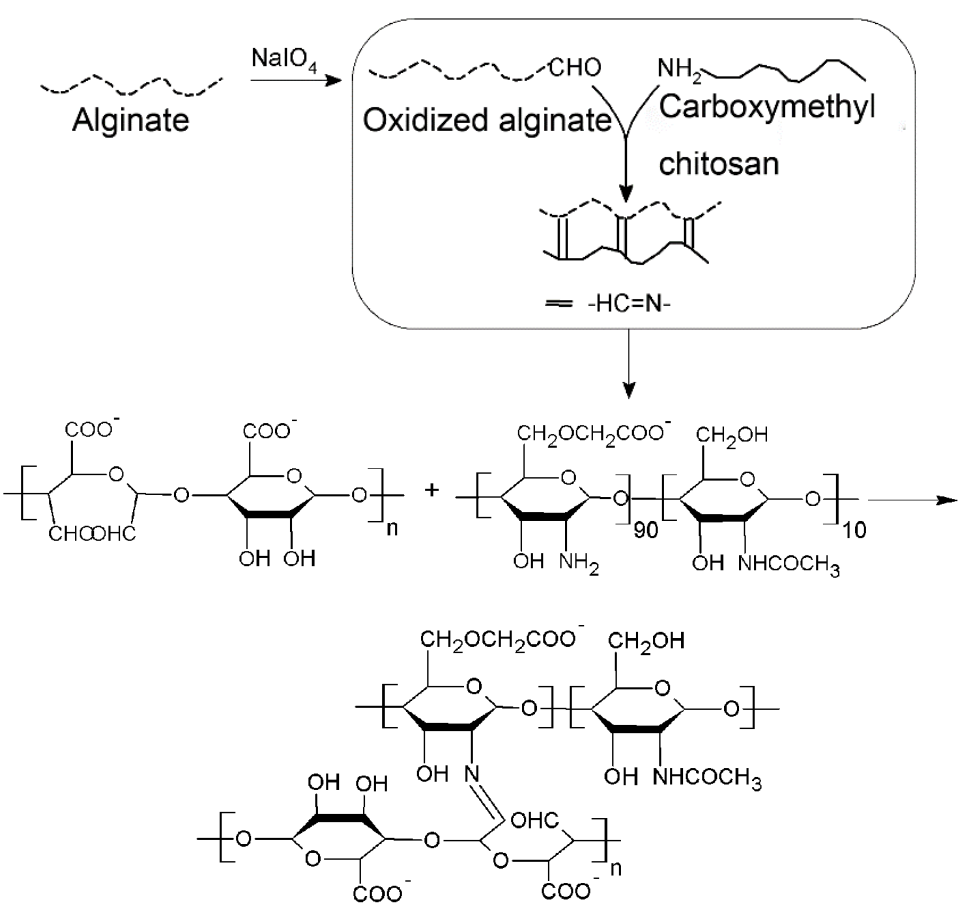

Fig. 2. Reaction mechanism of $C M C$ and OSA

\section{EXPERIMENTAL}

\section{Materials}

This study used analytically pure TEMPO (Sigma-Aldrich, Saint Louis,MO, USA), sodium hypochlorite $(\mathrm{NaClO})$, sodium chlorite $\left(\mathrm{NaClO}_{2}\right)$, sodium bromide $(\mathrm{NaBr})$, and absolute ethyl alcohol (Sinopharm Chemical Reagent Co., Ltd., Beijing, China). Lotus fibers were collected in Shouguang City, Shandong Province, China. The lotus species was Nelumbo nucifera, of the family Nymphaeaceae. After washing fresh lotus petiole, a ring was cut on its surface, and lotus fiber was obtained after breaking the material by hand. The fibers were placed in a beaker and dried at room temperature. Oxidized sodium alginate (self-made, DO of 9.3\%), and carboxymethyl chitosan (degree of substitution of $90 \%, M_{\mathrm{w}}=2.1 \times 10^{5}, M_{\mathrm{w}} / M_{\mathrm{n}}=3.2$, Qingdao Honghai Bio-Tech Co., Ltd., Beijing, China) were used. 


\section{Preparation and Characterization of NF}

Lotus fibers were purified by eliminating lignin and hemicellulose as described by He et al. (2013). $\mathrm{NaClO}_{2}(6.7 \mathrm{~g})$ was added to $700 \mathrm{~mL}$ of deionized water. The lotus fibers were evenly mixed with the $\mathrm{NaClO}_{2}$ solution (HJ-6A type, Jintan Medical Instrument Plant, Jintan, China). The solution was heated to $70{ }^{\circ} \mathrm{C}$ in a water bath. Glacial acetic acid $(1 \mathrm{~mL})$ was added every hour. The lotus fibers were placed in a sand-core funnel and cleaned with deionized water until the waste solution became neutral. Purified lotus fibers were mixed with $700 \mathrm{~mL}$ of $6 \% \mathrm{KOH}$ solution. The mixture was incubated at room temperature for $8 \mathrm{~h}$ and then heated in a water bath at $80{ }^{\circ} \mathrm{C}$ for $2 \mathrm{~h}$. The purified lotus fibers were placed in a sand-core funnel and washed with deionized water until the $\mathrm{pH}$ of the solution became neutral.

The pre-treated lotus fibers $(1 \mathrm{~g})$ were mixed with $100 \mathrm{~mL}$ of water. The solution was evenly mixed using a magnetic stirrer. TEMPO $(0.016 \mathrm{~g})$ and $\mathrm{NaBr}(0.1 \mathrm{~g})$ were added. After homogeneous dispersion, $\mathrm{NaClO}$ and $\mathrm{NaOH}$ were added to maintain a $\mathrm{pH}$ of 10 . At different reaction times at room temperature $(20,40,60,80$, and $100 \mathrm{~min})$ or when the $\mathrm{pH}$ value became stable, $5 \mathrm{~mL}$ of ethyl alcohol was added to terminate the reaction. The solution was centrifuged six times with deionized water (TG16, Changsha Yingtai Instrument Co., Ltd., Changsha, China) to remove the residues. The NF were obtained via the freeze-casting technology (LGJ-10, Beijing Songyuan Huaxing Technology Development Co., Ltd., Beijing, China). The chemical components of the NF were analyzed via the KBr pellet method and FTIR (Fourier Transform Infrared Spectroscopy). The NF yield was calculated using Eq. 2,

$$
Y=\left(W_{\mathrm{t}} / W_{0}\right) \times 100 \%
$$

where $Y$ is the NF yield, $W_{0}$ is the weight of lotus fibers, $W_{\mathrm{t}}$ is the weight of NF, and $Y, W_{0}$, and $W_{\mathrm{t}}$ are expressed in grams.

\section{Morphological Observation of NF}

A small amount of NF powder was mixed in water to produce the dispersion liquid with a mass percentage of $0.01 \%$. The NF dispersion liquid was subjected to ultrasonic dispersion for $20 \mathrm{~min}$ and stored for further use. The powders were obtained using a copper wire mesh loaded with a carbon film. After volatilization, the specimens were air-dried. The morphology of the NF was observed via transmission electron microscopy (TEM; model $\mathrm{H}$ - 7650, Hitachi, Tokyo, Japan) at a working voltage of $200 \mathrm{keV}$. The TEM images of NF were analyzed using image processing software, and the ratio of their length and diameter was calculated.

\section{DO Examination of NF}

At a ratio of 1:30, the oxidation products were immersed in $0.1 \mathrm{~mol} / \mathrm{L} \mathrm{HCl}$ for 40 min and washed with deionized water to completely remove chloridion. $\mathrm{AgNO}_{3}$ solution $(0.1 \mathrm{~mol} / \mathrm{L})$ was used to detect the presence of chloridion until no white precipitate was observed. The specimens were processed via freeze-casting technology and preserved in the dryer. The dry specimens were immersed in $50 \mathrm{~mL}$ of $2 \%$ calcium acetate solution. The solution was left to stand for $15 \mathrm{~h}$ with frequent shaking. Phenolphthalein indicators (100 $\mu \mathrm{L}$ ) with a mass percentage of $1 \%$ were added. Next, $0.1 \mathrm{~mol} / \mathrm{L}$ of $\mathrm{NaOH}$ standard solution was incrementally added until a light red color appeared for $30 \mathrm{~s}$. The consumed volume of $\mathrm{NaOH}$ was recorded. The degree of oxidation (DO) was calculated using Eq. 3, 


$$
D O=[(N \times V \times M \mathrm{~W}-\mathrm{COOH}) / m] \times 100 \%
$$

where $N$ is the molar concentration of the sodium hydroxide solution, $V$ is the consumed volume of the sodium hydroxide solution used for the titration of the specimens $(\mathrm{mL}), M_{\mathrm{W}-}$ $\mathrm{COOH}$ is the mmol mass of carboxyl, which is equal to $0.045 \mathrm{~g} / \mathrm{mmoL}$, and $m$ is the mass of the titrated specimens $(\mathrm{g})$.

\section{Degradation Test of COSA}

With $M_{\mathrm{w}}=3.0 \times 10^{5}$ as the base membrane, the sodium periodate oxidization method was used to prepare OSA (DO of 9.3\%). The OSA solution and carboxymethyl chitosan solution were then prepared. The two kinds of solutions were mixed evenly and placed in $37{ }^{\circ} \mathrm{C}$ until a gel was formed. The solution was pre-frozen at $-10^{\circ} \mathrm{C}$ for $12 \mathrm{~h}$ and then removed in the freeze-casting machine. After coating via ion sputtering, the solution was placed under a scanning electron microscope to visualize the microstructure. Solutions were marked as COSA-1, COSA-2, and COSA-3 based on the molar ratios of carboxymethyl chitosan and OSA in the solution $(1 / 1,2 / 1$, and 3/1), respectively.

The characterization of degradation performance was conducted as follows. The constant weights of COSA $\left(\mathrm{W}_{1}\right)$ were measured. They were immersed in ethyl alcohol with a volume fraction of 0.75 for 30 min. Phosphate buffer solution (PBS, pH 7.40) was used to remove ethyl alcohol. For the in vitro hydrolysis experiment, the COSA were then placed in the buffer with $1 \mathrm{mg} / \mathrm{mL}$ lysozyme ( $\mathrm{pH} 7.40$ ) and cultured in the incubator at $37{ }^{\circ} \mathrm{C}$ for two weeks. Three COSA were categorized into one group. A group was taken at setting time, centrifuged, and cleansed with deionized water. Finally, they were dried in a vacuum to achieve constant weight $\left(W_{2}\right)$.

\section{Degradation rates analysis}

The degradation rates were calculated using Eq. 4,

$$
\text { Degradation rate }=\frac{\left(W_{1}-W_{2}\right)}{W_{1}} \times 100 \%
$$

Testing the $\mathrm{pH}$ value of the degradation fluid

The $\mathrm{pH}$ value of the degradation fluid was detected using a precision acidity meter.

\section{Characterization of the chemical structure before and after degradation}

The chemical structure of the specimens before and after degradation was characterized by Fourier infrared spectrometer (Nicolet 5700, thermoelectric). It consists of Michelson interferometer and a computer. The main function of a Michelson interferometer is to make the light emitted by the light source divided into two beams to form a certain optical path difference, and then combine them to produce interference. The interference graph function obtained contains all frequency and intensity information of the light source. The Fourier transform of the interferogram function can be used to calculate the intensity distribution of the original light source in accordance with the frequency. The wavenumber range of 4000 to $400 \mathrm{~cm}^{-1}$ with the resolution of $4 \mathrm{~cm}^{-1}$ was used in this research.

\section{Chemical Crosslinking and the Performance of AFC Membranes}

AFC reprsented the lotus nanofibers/alginate porous membranes crosslinked with carboxymethyl chitosan. Lotus nanofibers (NF) with a DO of $9.7 \%$ suspension were mixed 
with OSA solution and carboxymethyl chitosan. At $37^{\circ} \mathrm{C}$, the mixture was gel-like during standing. The specimens were pre-frozen for $12 \mathrm{~h}$ at $-10{ }^{\circ} \mathrm{C}$. The mixture proceeded via freeze-casting technology into cellular porous membranes. The carboxymethyl chitosan and OSA were prepared with a molar ratio of 2:1. Based on the mass percent of lotus nanofibers $(0.02 \%, 0.03 \%, 0.04 \%, 0.05 \%$, and $0.06 \%)$, the porous membranes were marked as AFC-2, AFC-3, AFC-4, AFC-5, and AFC-6, respectively.

Properties of the membranes were evaluated using the $\mathrm{KBr}$ pellet technique, SEM, the liquid displacement method (Karageorgiou and Kaplan 2005), the water absorbency test (Kim et al. 2005), and tensile tests (ISO 527-3 1995). The above methods were applied in degradation tests of COSA to partly characterize effects on the chemical components, morphologic observation, porosity, water absorbency, tensile property, and degradation performance of AFC.

\section{RESULTS AND DISCUSSIONS}

\section{Relations between Reaction Time and NF Yield}

This research investigated the effects of different reaction times in the oxidation process on NF yield. The NF yields at different reaction times of 20, 40, 60, 80, and 100 min were $80.6 \%, 75.6 \%, 67.8 \%, 66.0 \%$, and $50.0 \%$, respectively. A slight increase in oxidation time led to a rapid decline in the yield, which indicated that the TEMPO oxidation system noticeably degraded lotus fibers. The relationship between the oxidation time and yield was consistent with findings in the literature, as TEMPO oxidation system causes significant cellulose degradation (Shibata and Isogai 2003).

\section{Effects of the Preparation Process on NF Morphology and the Length- Diameter Ratio}

The macro-morphology of lotus fibers (Fig. 3a) before and after oxidation changed greatly. The NFs were obtained from the oxidation of lotus fibers by using the TEMPO/NaClO/NaBr system. They were processed via centrifugal washing and yielded a suspension characterized by a translucent paste (Fig. 3b).

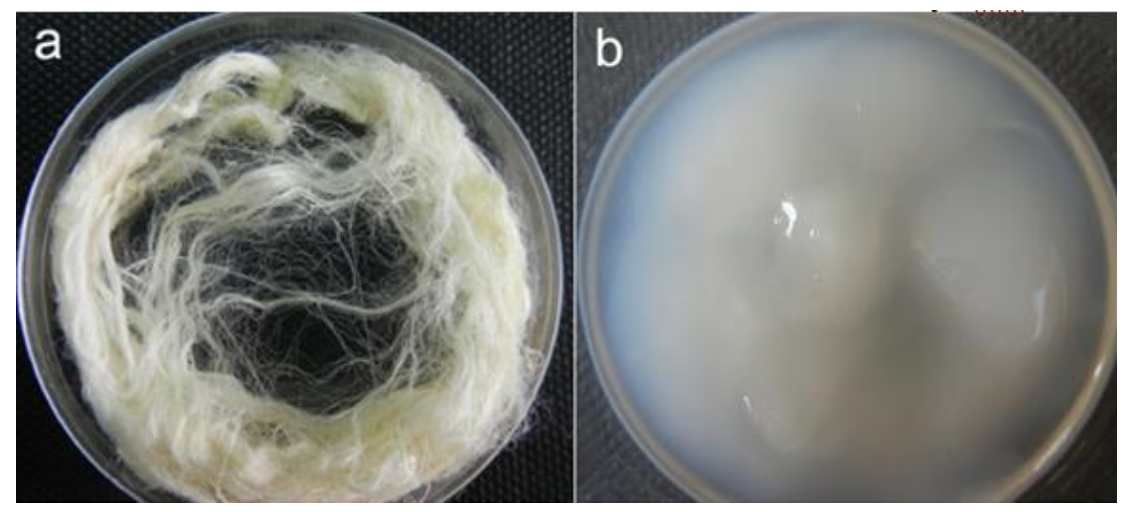

Fig. 3. Morphology of (a) lotus fibers and (b) NF

Obtaining NF via the lotus fibers oxidation was correlated to the degradation effects of the TEMPO system on cellulose. The photos were analyzed by Image-Pro-Plus image software on physical characteristics, then lotus fiber's diameter were obtained. Partial lotus 
fibers were dissolved and removed from the oxidation process. The damage in the amorphous region led to the dissolution of a portion of the celluloses. The formation of the hydrogen bond between the hydroxyl and carboxyl groups in the NF and water also caused the degradation of celluloses. The degradation of celluloses led to the formation of a 3D network structure and the enhancement of viscosity (Bai et al. 2009).

The diameter of lotus fibers was approximately $3 \mu \mathrm{m}$ (Pan et al. 2011). Figure 4a shows that the diameter of lotus fibers decreased to $2.0 \mu \mathrm{m}$ after pre-processing because of the removal of hemicellulose, lignin, and other components, which also reduced the diameter of fibers. Figure $4 \mathrm{~b}$ shows that a small amount of NF was produced after $40 \mathrm{~min}$ of oxidation. The length and diameter of NF were measured as $403 \mathrm{~nm}$ and $15 \mathrm{~nm}$, respectively, and the length-diameter ratio exceeded 25:1. Figure 4c shows that the diameter of NF oxidized for $60 \mathrm{~min}$ was $15 \mathrm{~nm}$ and the length-diameter ratio exceeded 1000:1. Acid soluble, lignin alkali-soluble lignin and hemicellulose were removed from lotus fibers by glacial acetic acid and $\mathrm{KOH}$. The amount noticeable exceeded NF after 40 min of oxidation, which was conducive for the enhancement of composite materials. Because lotus fibers are superfine, the oxidation time of NF prepared via TEMPO/NaClO/NaBr oxidation was remarkable less compared with that of cotton nanofibers (Missoum et al. 2013).
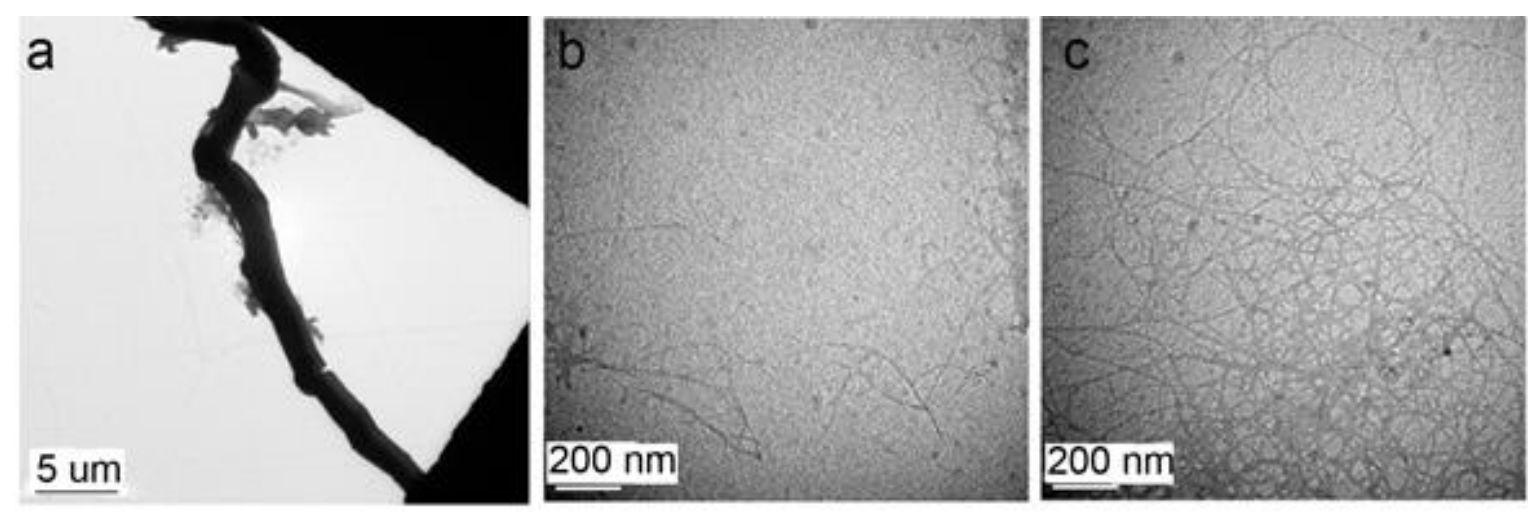

Fig. 4. TEM of (a) pretreated lotus fibers, (b) NF of 40 min of oxidation, and (c) NF of 60 min of oxidation

\section{Chemical Composition of NF}

After pre-processing, the characteristic peak of lignin in the lotus fibers (Fig. 5a LF) disappeared at 1509 and $1593 \mathrm{~cm}^{-1}$ (as shown in the Fig. 5a PLF), and the absorption peak did not appear at $1460 \mathrm{~cm}^{-1}$ (Bismarck et al. 2001). 

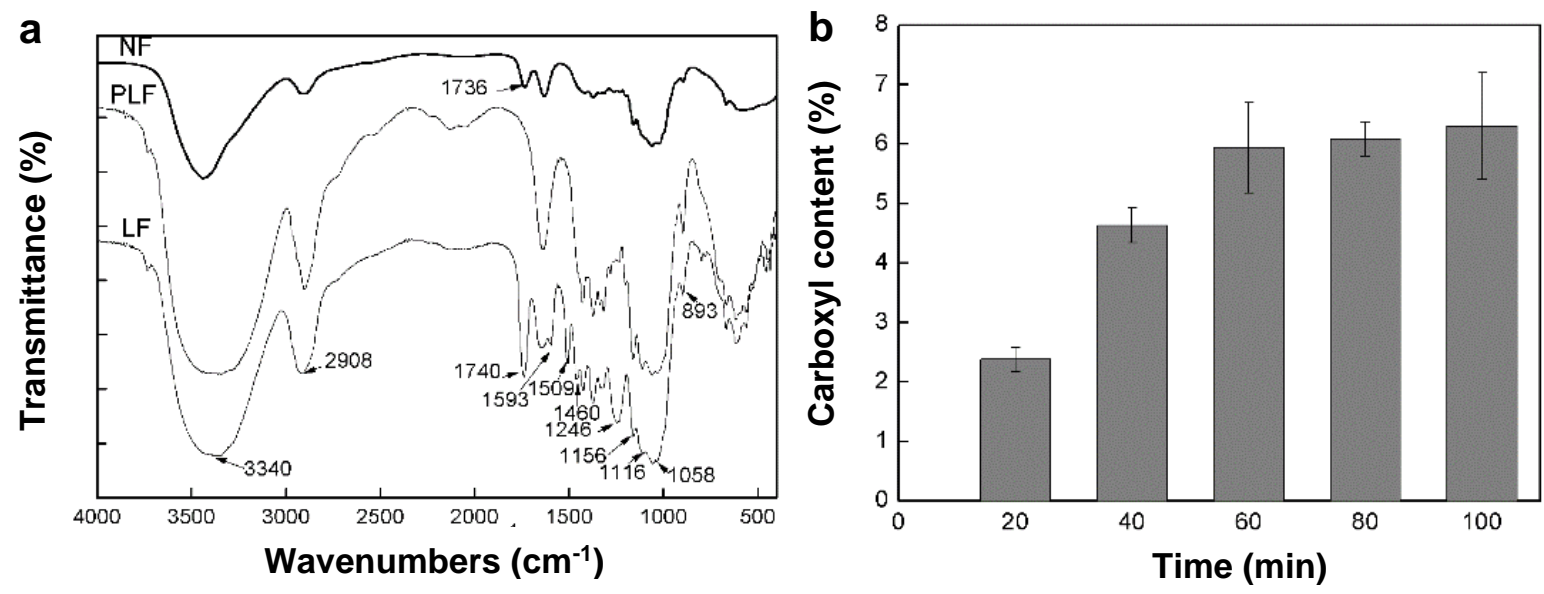

Fig. 5. (a) FTIR spectra changes of lotus fibers pre and post oxidation and (b) Relationship between $\mathrm{DO}$ of $\mathrm{NF}$ and oxidation time

The results indicated the removal of hemicellulose and lignin; the cellulose was preserved. Oxidation reaction in the preparation of NF (Fig. 5a NF) was conducted at alkaline conditions. To avoid the interference of the peak for water near $1630 \mathrm{~cm}^{-1}$, the samples were prepared after neutralization by using $\mathrm{HCl}$. At $1736 \mathrm{~cm}^{-1}$, the characteristic absorption peak of carboxyl appeared. The FTIR results show that carboxyl was introduced to the molecular chain of the lotus fibers after the successful TEMPO/ $\mathrm{NaClO} / \mathrm{NaBr}$ oxidation.

\section{Influencing Patterns of Reaction Conditions on DO of NF}

When the usage of TEMPO, $\mathrm{NaClO}$, and $\mathrm{NaBr}$ was certain, the $\mathrm{pH}$ values of the reaction solution after $100 \mathrm{~min}$ of reaction remained unchanged, which indicated the termination of the reaction. In Fig. 5b, increasing the reaction time led to an increase in the DO. A long reaction time leads to the frequent occurrence of oxidation reactions. As such, the DO increased. During the reaction, the reaction time was extended and the amount of peeled glucose increased (Qian et al. 2010). Thus, the time of oxidation reaction should be controlled. The termination of the oxidation reaction can be accomplished in a short time by using lotus fibers as the raw material, and this result provides a theoretical basis for energy saving in the industrial production of nanocellulose.

\section{Degradation Performance of COSA \\ Degradation rates}

The surface (Fig. 6a) and the cross section (Fig. 6b) of the porous membrane had relatively more polygonal pores. The protrusions on the surface of COSA facilitated cell adhesion. The polygonal pores were also intersected. The formed network structure was compact, and the range of pore diameter ranged from $100 \mu \mathrm{m}$ to $200 \mu \mathrm{m}$ (Vieira et al. 2008). The porous alginate membrane can be prepared via chemical crosslinking and single freeze-drying. As such, the energy consumption and preparation time for COSA were significantly decreased. The CMC molecular chains had substantial amino, hydroxyl, and carboxyl content. The OSA molecular chains also had aldehyde, hydroxyl, and carboxyl groups. When the CMC and OSA were mixed, Schiff base and hydrogen bonds were produced. In addition, the intertwining of molecules also turned the mixed solution into a gel. 

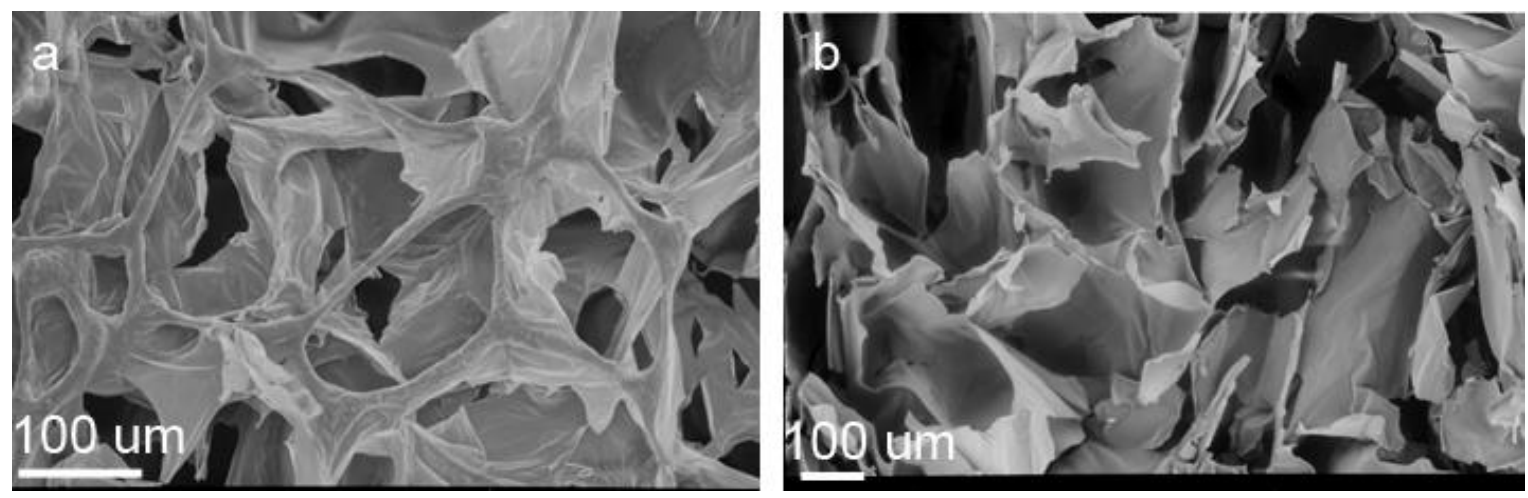

Fig. 6. SEM of COSA (a) surface and (b) cross section

The membranes with a molar ratio of 2/1 (COSA-2) showed the longest time of stability (Fig. 7a). After two d of degradation, the mass was quickly reduced by $20 \%$. Subsequently, the declining rate was slower. At $14 \mathrm{~d}, 82.1 \%$ of the membrane was degraded. The decline in the degradation rate of COSA-2 was slower because the reaction molar ratio between OSA and CMC was a suitable theoretical value. Their crosslinking produced sufficient Schiff base chemical bonds and hydrogen bonds. The OSA carboxyl with a negative charge exhibited an electrostatic attraction with the CMC carrying an amino group. The electrostatic interaction resulted in a stable 3D structure that endured degradation. This membrane is expected to be used in the medical field. The results indicated that adjusting the molar ratio between OSA aldehyde and CMC amino could regulate the degradation rates of the porous membranes (Li et al. 2012).

\section{Variation patterns in the $\mathrm{pH}$ value of the degradation liquid}

The $\mathrm{pH}$ value of the degradation liquid of COSA-2 and COSA-3 showed an overall rising trend (Fig. 7b).

In the first two $d$ of degradation, the rate of increase was rapid and followed by a slight decline. After $14 \mathrm{~d}$ of degradation, the $\mathrm{pH}$ values of COSA-2 and COSA-3 were 7.5 and 7.6, respectively, because the high molar ratio of CMC. After CMC degradation, the amino glucose molecules or oligomers and other degradation products containing amino were produced. The products were alkaline, which led to an increase in the $\mathrm{pH}$ value of the degradation liquid. The OSA degradation reduced the $\mathrm{pH}$ value of the degradation liquid. The membrane with a molar ratio of $1 / 1$ was influenced by OSA, which caused a decrease in the $\mathrm{pH}$ value. 




a
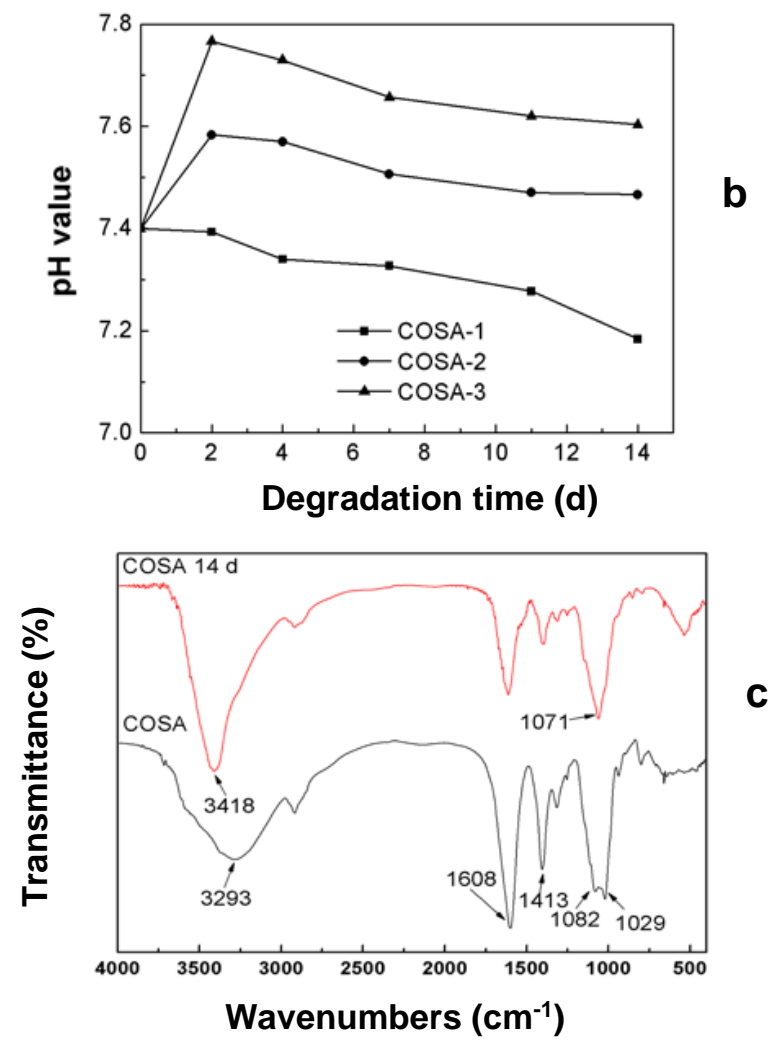

Fig. 7. (a) Degradation rates of COSA during $14 \mathrm{~d}$. (b) $\mathrm{pH}$ values of degradation solutions during degradation. (c) FTIR spectral changes of COSA

\section{Infrared spectroscopy analysis}

The peak of degraded COSA at $3418 \mathrm{~cm}^{-1}$ was noticeably narrowed prior to degradation (Fig. 7c), which indicated a decrease in the number of hydrogen bonds from $\mathrm{O}-\mathrm{H}$. The peak intensity at 1608 and $1413 \mathrm{~cm}^{-1}$ was noticeably weakened, and the peak shape at $1071 \mathrm{~cm}^{-1}$ was sharpened. The weakened intensity and sharpened peak shape of COSA indicated that the macromolecular chain of COSA was cut off, the gap between molecules was enhanced, and the internal structure was loosened. The binding force between the segments of the molecular chain was also weakened, which indicated that lysozyme influenced the macromolecular chain of CMC through an internally tangent approach. This led to the breakage of the $\beta$-1,4-glycosidic bond and the degradation of CMC. 
Overall, a small oxidation ratio of OSA-10 (DO as 9.3\%) resulted in a limited amount of Schiff base chemical bonds formed with CMC. Most of the COSA-2 was degraded after $14 \mathrm{~d}$. However, compared with COSA-1 and COSA-3, COSA-2 was the most stable during degradation.

\section{AFC Microstructure}

Lotus nanofibers (NF) and OSA solution were crosslinked via CMC. These three macromolecular chains intertwined to form a three-dimensional network structure. Figure 8 shows an AFC macroscopic morphology prepared via CMC crosslinking. The membranes produced via the freeze-drying method were white, soft, and difficult to break. The membrane surface and the pores of the cross section were cellular-shaped (Fig. 9). The pores were arranged in an orderly way, and they were interlaced with many links.
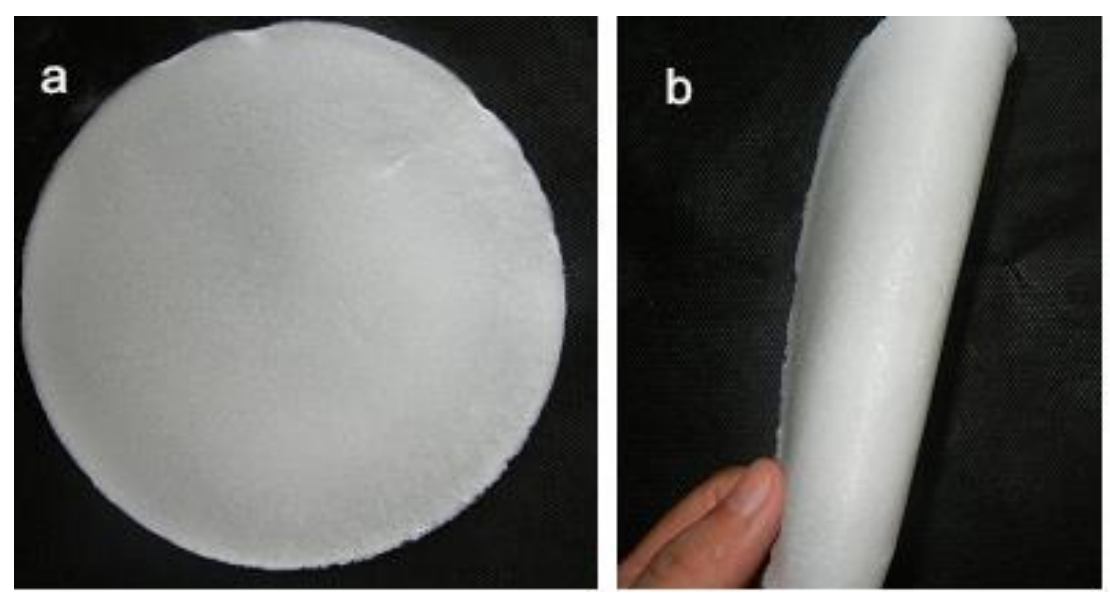

Fig. 8. Macroscopic photos of AFC: (a) surface; (b) flexed AFC
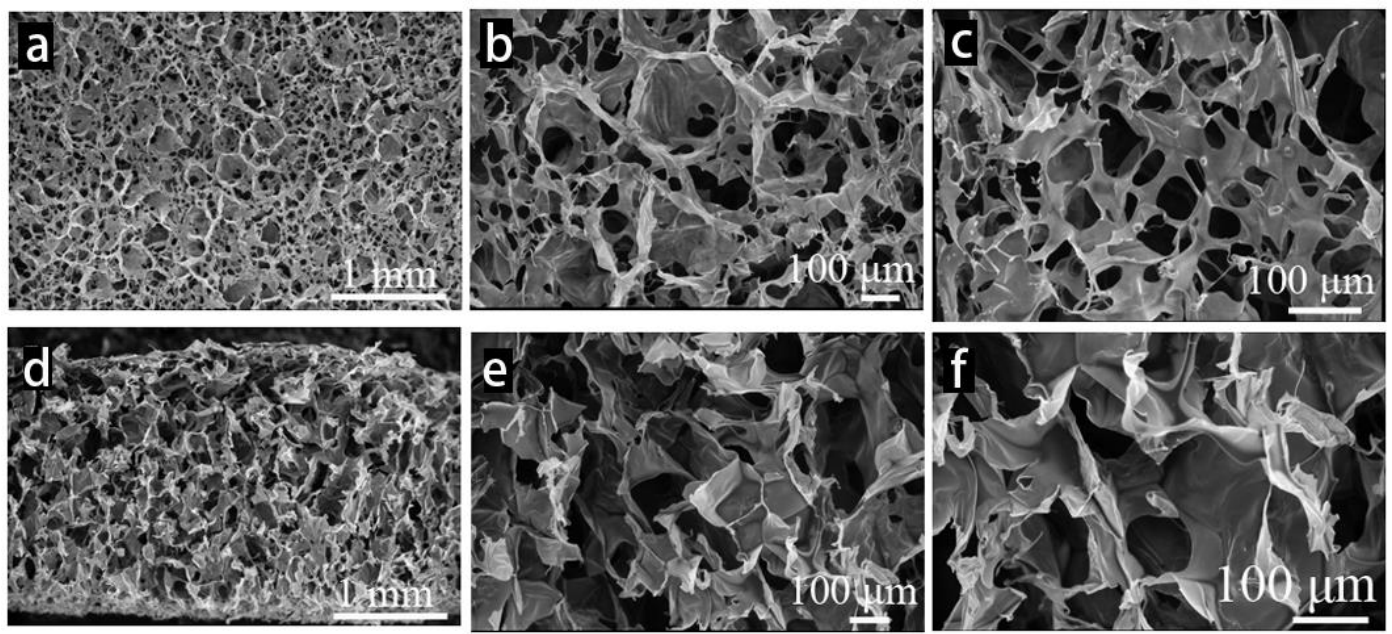

Fig. 9. SEM images of AFC: (a, b, and c) surface; (d, e, and f) cross section

\section{Analysis of AFC Tensile Property}

The strength of biological membranes is crucial because they provide support for new tissues and space for cell growth until new tissues develop biomechanical characteristics. The strength is expected to match the degradation rate. As shown in Fig. 
$10 \mathrm{a}$, the tensile strength and elongation at the break of the porous membranes added with traces of NF were better than those of the alginate porous membranes without NF. The elongation at the break was $5.1 \%$ to $7.7 \%$. The values were close to the elongation at the break $(9.5 \%)$ of the human skin. When the NF amount was increased, the tensile strength was enhanced, which indicated that AFC had good tensile strength.
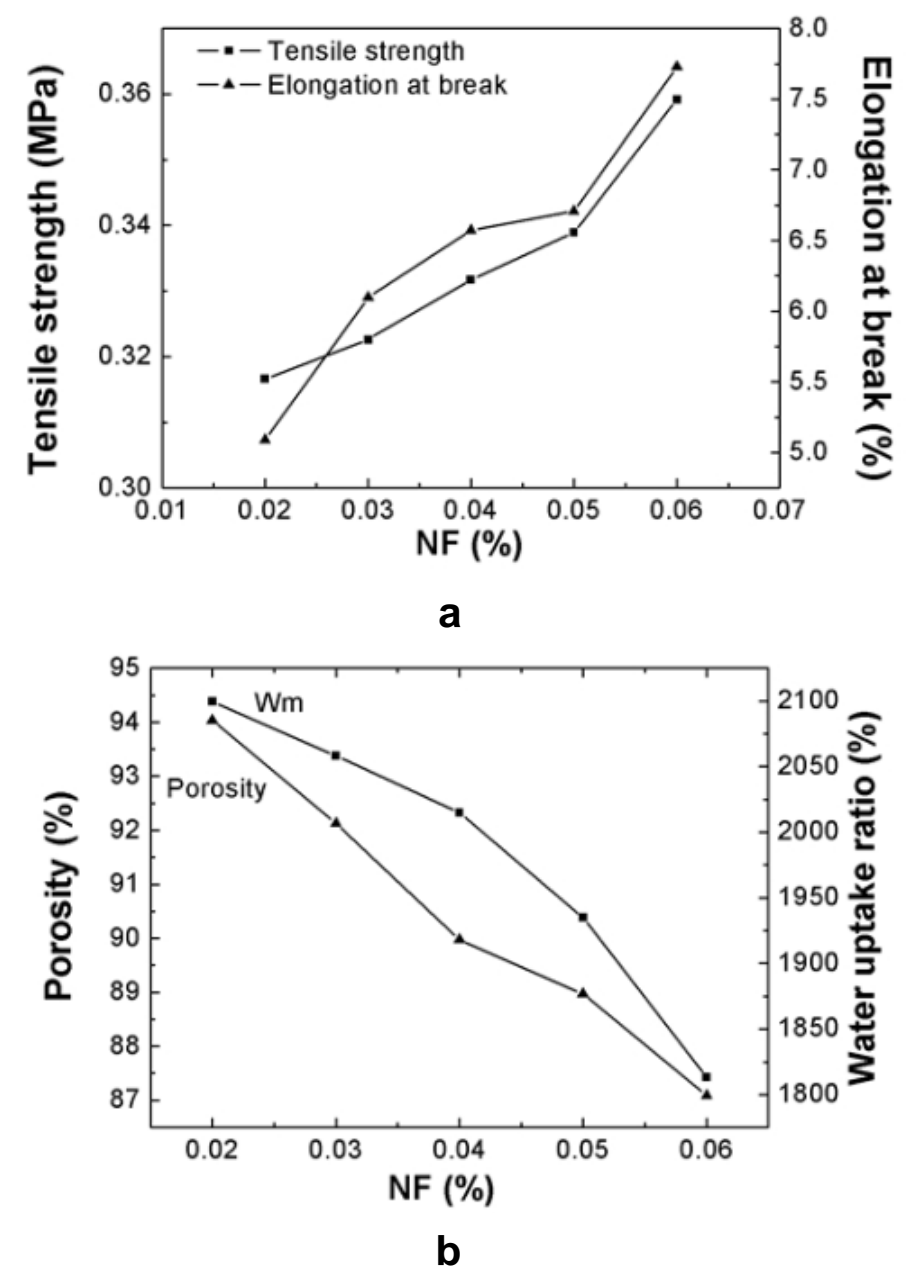

Fig. 10. Effect of NF dosage on (a) tensile property and elongation at the break of AFC and (b) porosity and water uptake ratio of AFC

Nanometer materials have a large proportion of atoms on the surface, which means that they have a very high specific surface and surface energy. Due to the increased number of surface atoms, insufficient coordination of atoms, and high surface energy, these surface atoms are highly active, unstable, and easy to combine with other atoms. For example, metal nanoparticles burn when exposed to air. Likewise, inorganic nanoparticles absorb gases and react with them when exposed to air. Similarly, it was found that the NFs were able to form more hydrogen bonds with carboxymethyl chitosan and oxidation sodium alginate. The increased number of hydrogen bonds reinforced the 3D network and tensile property of AFC. The negative charge carried by carboxyl groups on the oxidized NF molecules and carboxymethyl chitosan carrying a positive charge formed ionic bonds, which also strengthened the 3D network. The ionic bond energy was stronger than hydrogen bond strength. The use of more NFs from the TEMPO oxidation resulted in more 
ionic and hydrogen bonds. Thus, better AFC tensile properties were obtained.

\section{AFC Porosity and Water Absorbency Analysis}

When the NF usage was increased from $0.02 \%$ to $0.06 \%$, the porosity of the porous membrane decreased from $94.0 \%$ to $87.1 \%$ (Fig. 10b). Generally, the porosity showed a gradual decreasing trend, and its mutual effects with the NF were enhanced (Lin et al. 2012). Moreover, the AFC structure became more compact. The porosity directly influenced water absorbency, tensile property, and degradation performance. When the NF usage was increased, water absorbency decreased from $2100 \%$ to $1810 \%$, which was consistent with decreased porosity. The spatial structure was also significantly affected by water absorbency. Membranes with a large porosity performed remarkably well in terms of water absorbency because the pores in the membrane functioned as entry points for water. The large porosity enabled a smoother water flow, which allowed the water to penetrate the membrane at a faster pace (Ninan et al. 2013).

\section{AFC Degradation Behaviors}

\section{AFC degradation rates}

The degradation rates of AFC were relatively large. After $14 \mathrm{~d}$ of degradation, the degradation ranged from $59.2 \%$ to $70.0 \%$. The NF was crosslinked with the OSA solution via $\mathrm{CMC}$. The three macromolecular chains intertwined to form a 3D network structure. The large degradation rates of carboxymethyl chitosan promoted AFC degradation. When the NF content was increased, the degradation rates of NF decreased. Moreover, the AFC degradation rates were reduced. Thus, after $14 \mathrm{~d}$ of degradation, the degradation rate of AFC-6 was smallest among that of AFC-2, AFC-4, and AFC-6. The rates of water immersion in the membranes were greater than the degradation rates of the membranes (Figs. 11a). Thus, AFC degradation is classified as bulk degradation (Fujii et al. 2013).

The $\mathrm{pH}$ value of the degradation liquid of $A F C$

At the initial stage of degradation, the $\mathrm{pH}$ value initially increased and subsequently declined (Fig. 11b). The increase in $\mathrm{pH}$ value can be attributed to the fast degradation of $\mathrm{CMC}$ under the effects of lysozyme. Its products can increase the $\mathrm{pH}$ value of the degradation liquid. The OSA and NF were then degraded and the $\mathrm{pH}$ value decreased. After acid-base neutralization, the $\mathrm{pH}$ value of the degradation liquid remained neutral, thereby facilitating the growth of human cells. The large degradation rate of AFC-2 produced a substantial amount of degradation products. The $\mathrm{pH}$ value (7.3) of the degradation liquid was attributed to the decreased NF content in the membranes. 


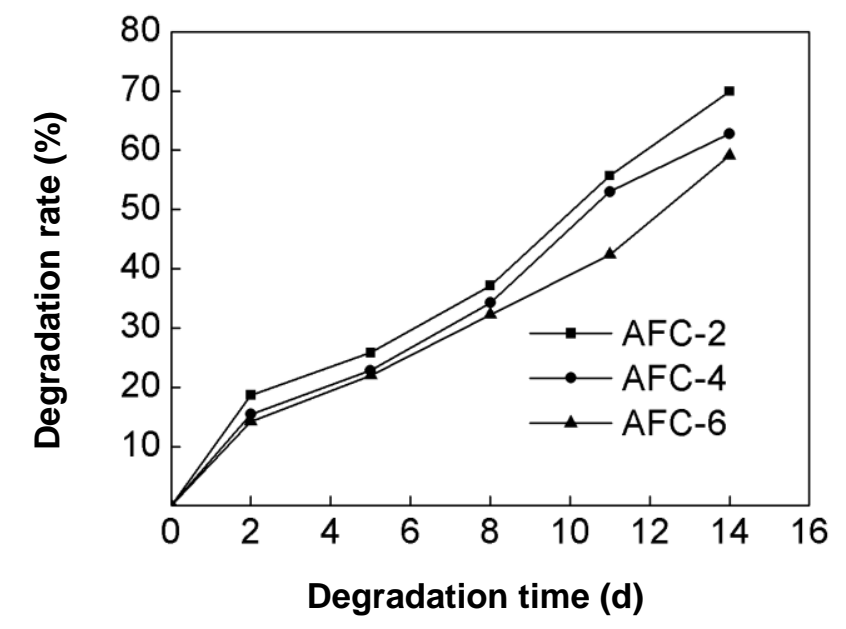

a

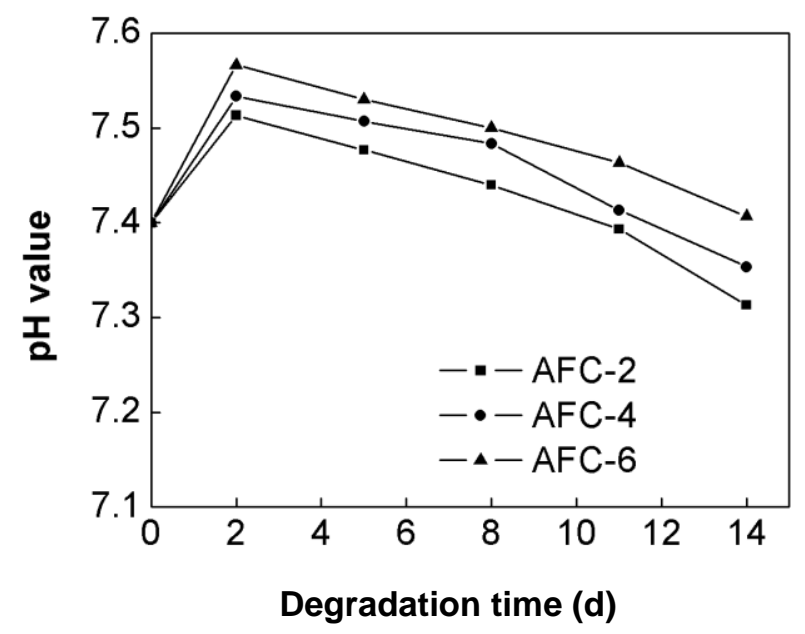

b

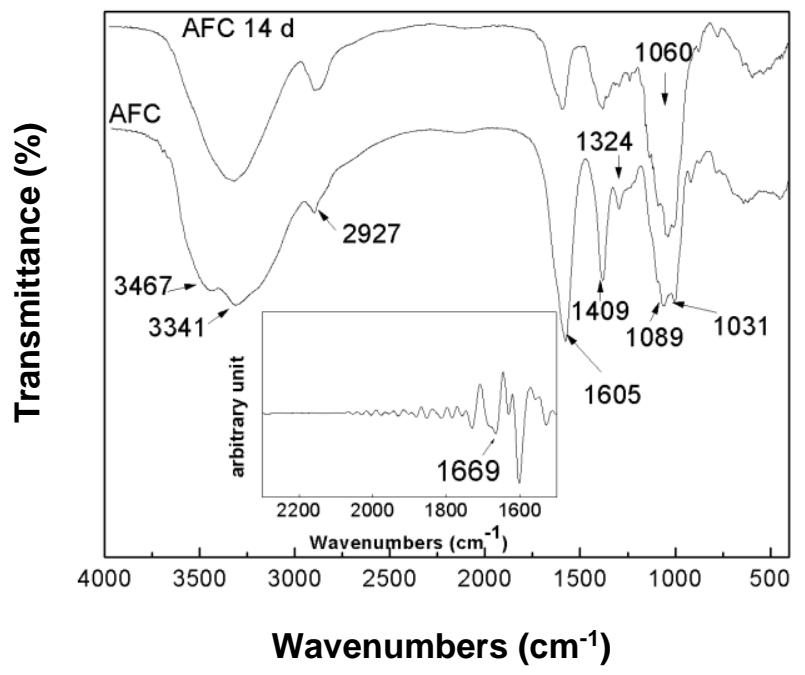

Fig. 11. (a) Degradation rates variations of $A F C$, (b) the $\mathrm{pH}$ variations of $A F C$ porous membranes, and (c) FTIR spectra of AFC before and after degradation 


\section{Infrared spectroscopy of AFC}

The absorption peaks of $\mathrm{N}-\mathrm{H}$ and $\mathrm{O}-\mathrm{H}$ bonds appeared at 3467 and $3341 \mathrm{~cm}^{-1}$ (Fig. 11c). The absorption peak of $\mathrm{C}-\mathrm{H}$ bond appeared near $2927 \mathrm{~cm}^{-1}$. The reaction of carboxymethyl chitosan and oxidation sodium alginate produced the Schiff base $(-\mathrm{C}=\mathrm{N}-)$ characteristic peak, which appeared at $1669 \mathrm{~cm}^{-1}$ of the derivate spectrum. Peaks near 1605 $\mathrm{cm}^{-1}$ were attributed to -COO- dissymmetrical stretching vibration and $\mathrm{N}-\mathrm{H}$ bending vibration peaks on the molecular chains of the OSA, CMC, and NF. Peaks near $1409 \mathrm{~cm}^{-1}$ were the $-\mathrm{COO}-$ symmetrical stretching vibration and $\mathrm{C}-\mathrm{H}$ absorption peak. Peaks near $1324 \mathrm{~cm}^{-1}$ were produced by $\mathrm{C}-\mathrm{H}$ vibration. Peaks near 1031 and $1089 \mathrm{~cm}^{-1}$ were $-\mathrm{C}-\mathrm{O}-$ stretching vibration peaks in the polysaccharide molecular structure of the above three polymers.

After $14 \mathrm{~d}$ of degradation, the peak intensity at 1605 and $1409 \mathrm{~cm}^{-1}$ was significantly weakened, which indicated that the molecular chains broke after degradation and that oligomers were formed. At the same time, the shape of $-\mathrm{C}-\mathrm{O}-$ stretching vibration peak near $1089 \mathrm{~cm}^{-1}$ changed. The change in the vibration peak verified that the hexatomic ring was also changed.

\section{CONCLUSIONS}

1. By regulating the preparation conditions, the degrees of oxidation (Dos) of nanofibers (NF) can be changed. The increased oxidation time led to an increase in the DOs.

2. Addition of carboxylmethyl cellulose (CMC) significantly improved the degradation performance of the porous membranes. Up to $82.1 \%$ of the COSA could be degraded within $14 \mathrm{~d}$, and the structure integration of CMC could be sufficiently maintained. When the molar ratio of $\mathrm{CMC}$ was increased, the $\mathrm{pH}$ value increased. Adjusting the molar ratio of the components regulated the degradation of the COSA.

3. The porous membranes (AFC) exhibited a relatively large porosity, high water absorbency, better tensile property, and excellent degradation performance.

\section{ACKNOWLEDGEMENTS}

The work was funded by a grant from the National Natural Science Foundation of China under Grant No. 51373083; the National Natural Science Foundation of Shandong Province, China under Grant No. ZR2012EMZ002; Shandong Province Young and Middle-Aged Scientists Research Awards Fund under Grant No. BS2013CL008; Qingdao Sci-Tech Planning Project under Grant No. 13-1-4-212-jch; and the Young Teacher Training Program for Innovation Team in Medical College of Qingdao University.

\section{REFERENCES CITED}

Bai, W., Holbery, J., and Li, K. (2009). "A technique for production of nanocrystalline cellulose with a narrow size distribution," Cellulose 16(3), 455-465. DOI: 10.1007/s10570-009-9277-1

Bidarra, S. J., Barrias, C. C., and Granja, P. L. (2014). "Injectable alginate hydrogels for cell delivery in tissue engineering," Acta Biomater. 10, 1646-1662. DOI: 
10.1016/j.actbio.2013.12.006

Bismarck, A., Mohanty, A. K., Aranberri-Askargorta, I., Czapla, S., Misra, M., Hinrichsen, G., and Springer, J. (2001). "Surface characterization of natural fibers; Surface properties and the water up-take behavior of modified sisal and coir fibers," Green Chem. 3(2), 100-107. DOI: 10.1039/b100365h

Fujii, S., Miyanari, Y., Nishimura, T., Yokoyama, Y., Hamasaki, S., Okada, M., Furuzono, T., Matsuda, S., Takamori, H., and Nakamura, Y. (2013). "In vitro degradation of hydroxyapatite nanoparticle-coated biodegradable microspheres," Polym. Degrad. Stabil. 98(1), 377-386. DOI: 10.1016/j.polymdegradstab.2012.09.003

Gao, C., Liu, M., Chen, J., and Chen, C. (2011). "Interactions between bovine serum albumin and oxidized sodium alginate in solution," J. Biomat. Sci.-Polym. E. 22, 1639-1650. DOI: 10.1163/092050610X519462

Gong, Y., Han, G. T., Zhang, Y. M., Pan, Y., Li, X., Xia, Y., and Wu, Y. (2012). "Antifungal activity and cytotoxicity of zinc, calcium, or copper alginate fibers," Biol. Trace Elem. Res. 148, 415-419. DOI: 10.1007/s12011-012-9388-7

He, W., Jiang, S., Zhang, Q., and Pan, M. (2013). "Isolation and characterization of cellulose nanofibers from Bambusa rigida," BioResources 8(4), 5678-5689. DOI: 10.15376/biores.8.4.5678-5689

Hennink, W., and Van Nostrum, C. (2012). "Novel crosslinking methods to design hydrogels," Adv. Drug Delivery Rev. 64, 223-236. DOI: 10.1016/j.addr.2012.09.009

ISO 527-3 (1995). "Plastics-determination of tensile properties-Part 3: Test conditions for films and sheets," International Organization for Standardization, Geneva, Switzerland.

Karageorgiou, V., and Kaplan, D. (2005). "Porosity of 3D biomaterial scaffolds and osteogenesis," Biomaterials 26, 5474-5491. DOI: 10.1016/j.biomaterials.2005.02.002

Kim, U. J., Park, J., Kim, H., Wada, M., and Kaplan, D. L. (2005). "Three-dimensional aqueous-derived biomaterial scaffolds from silk fibroin," Biomaterials 26, 27752785. DOI: 10.1016/j.biomaterials.2004.07.044

Khil, M. S., Kim, H. Y., Kang, Y. S., Bang, H. J., Lee, D. R., and Doo, J. K. (2005). "Preparation of electrospun oxidized cellulose mats and their in vitro degradation behavior," Macromol. Res. 13(1), 62-67. DOI: 10.1007/BF03219016

Lee, K. Y., and Mooney, D. J. (2012). “Alginate: Properties and biomedical applications," Prog. Polym. Sci. 37(1), 106-126. DOI:

10.1016/j.progpolymsci.2011.06.003

Lin, N., Bruzzese, C., and Dufresne, A. (2012). "TEMPO-oxidized nanocellulose participating as crosslinking aid for alginate-based sponges," ACS Appl. Mater. Interface. 4, 4948-4959. DOI: 10.1021/am301325r

Li, X., Weng, Y., Kong, X., Zhang, B., Li, M., Diao, K., Zhang, Z., Wang, X., and Chen, H. (2012). "A covalently crosslinked polysaccharide hydrogel for potential applications in drug delivery and tissue engineering," J. Mater. Sci.-Mater. M. 23, 2857-2865. DOI: 10.1007/s10856-012-4757-5

Missoum, K., Belgacem, M. N., and Bras, J. (2013). "Nanofibrillated cellulose surface modification: A review," Materials 6(5), 1745-1766. DOI: 10.3390/ma6051745

Ninan, N., Muthiah, M., Park, I.-K., Elain, A., Thomas, S., and Grohens, Y. (2013). "Pectin/carboxymethyl cellulose/microfibrillated cellulose composite scaffolds for tissue engineering," Carbohyd. Polym. 98(1), 877-885. DOI: 10.1016/j.carbpol.2013.06.067

Pan, Y., Han, G., Mao, Z., Zhang, Y., Duan, H., Huang, J., and Qu, L. (2011). "Structural 
characteristics and physical properties of lotus fibers obtained from Nelumbo nucifera petioles," Carbohyd. Polym. 85(1), 188-195. DOI: 10.1016/j.carbpol.2011.02.013

Pantaleone, D., Yalpani, M., and Scollar, M. (1992). "Unusual susceptibility of chitosan to enzymic hydrolysis," Carbohyd. Res. 237, 325-332. DOI: 10.1016/S00086215(92)84256-R

Pawar, S. N., and Edgar, K. J. (2012). "Alginate derivatization: A review of chemistry, properties and applications," Biomaterials 33, 3279-3305. DOI: 10.1016/j.biomaterials.2012.01.007

Peng, S., Zheng, Y., Wu, J., Wu, Y., Ma, Y., Song, W., and Xi, T. (2012). "Preparation and characterization of degradable oxidized bacterial cellulose reacted with nitrogen dioxide," Polym. Bull. 68(2), 415-423. DOI: 10.1007/s00289-011-0550-8

Qian, R. J., Tang, A. M., Song, J. K. (2010). "Effect of TEMPO selective oxidation on cellulose-morphology and paper properties," Paper and Paper Making 29(10), 22-25. DOI: $10.13472 /$ j.ppm.2010.10.010

Rowland, C. R., Lennon, D. P., Caplan, A. I., and Guilak, F. (2013). "The effects of crosslinking of scaffolds engineered from cartilage ECM on the chondrogenic differentiation of MSCs," Biomaterials 34, 5802-5812. DOI: 10.1016/j.biomaterials.2013.04.027

Souguir, Z., Picton, L., and Le Cerf, D. (2012). "Anionic polysaccharide hydrogels with charges provided by the polysaccharide or the crosslinking agent," Drug Deliv. Lett. 2, 240-250. DOI: 10.2174/2210304x11202040002

Shibata, I., and Isogai, A. (2003). "Depolymerization of cellouronic acid during TEMPOmediated oxidation," Cellulose 10(2), 151-158. DOI: 10.1023/a:1024051514026

Vieira, E. F., Cestari, A. R., Airoldi, C., and Loh, W. (2008). "Polysaccharide-based hydrogels: Preparation, characterization, and drug interaction behaviour," Biomacromolecules 9, 1195-1199. DOI: 10.1021/bm7011983

Wu, Y., He, J., Cheng, W., Gu, H., Guo, Z., Gao, S., and Huang, Y. (2012). “Oxidized regenerated cellulose-based hemostat with microscopically gradient structure," Carbohyd. Polym. 88(2012), 1023-1032. DOI: 10.1016/j.carbpol.2012.01.058

Article submitted: December 3, 2018; Peer review completed: May 3, 2020; Revised version received and accepted: June 27, 2020; Published: July 6, 2020.

DOI: 10.15376/biores.15.3.6471-6487 